\title{
Technofixing the Future: Ethical Side Effects of Using AI and Big Data to meet the SDGs
}

\author{
Mark Ryan \\ Department of Philosophy, \\ University of Twente \\ Enschede, the Netherlands \\ m.j.ryan@utwente.nl
}

\author{
Josephina Antoniou \\ School of Sciences \\ University of Central Lancashire, \\ Larnaka, Cyprus \\ JAntoniou@uclan.ac.uk \\ Kevin Macnish \\ Department of Philosophy, \\ University of Twente \\ Enschede, the Netherlands \\ k.macnish@utwente.nl
}

\author{
Laurence Brooks \\ School of Computer Science and \\ Informatics, De Montford \\ University, United Kingdom \\ laurence.brooks@dmu.ac.uk \\ Bernd Stahl \\ School of Computer Science and \\ Informatics, De Montford \\ University, United Kingdom \\ b.stahl@utwente.nl
}

\author{
Tilimbe Jiya \\ School of Computer Science and \\ Informatics, De Montford \\ University, United Kingdom \\ tilimbe.jiya@dmu.ac.uk
}

\begin{abstract}
-while the use of smart information systems (the combination of $\mathrm{AI}$ and Big Data) offer great potential for meeting many of the UN's Sustainable Development Goals (SDGs), they also raise a number of ethical challenges in their implementation. Through the use of six empirical case studies, this paper will examine potential ethical issues relating to use of SIS to meet the challenges in six of the SDGs $(2,3,7,8,11$, and 12). The paper will show that often a simple "technofix", such as through the use of SIS, is not sufficient and may exacerbate, or create new, issues for the development community using SIS.
\end{abstract}

Keywords-artificial intelligence, Big Data, sustainable development goals, ethics, smart information systems

\section{INTRODUCTION}

Smart information systems (SIS), those technologies that build on big data analytics, typically facilitated by machine learning implemented through deep neural networks [1], are expected to grow in importance and impact. Some of these impacts are likely to be beneficial, notably the growth in efficiency and profits, which will contribute to societal wellbeing [2]. Beyond purely economic benefits, SIS can be used to address global challenges, such as those outlined in UN's Sustainable Development Goals (SDGs). SIS hold great potential to increase crop yields, expose discrimination, reduce pollution and improve the infrastructure, amenities, and livability of cities; closely aligning with many of the aims of the SDGs.

At the same time, these technologies can raise significant worries and ethical concerns, such as algorithmic bias, job loss, power asymmetries, privacy infringements, and surveillance. SIS also have the potential to exacerbate inequality and further entrench the market dominance of big tech companies, if left unregulated. If SIS are not used responsibly, they may not only be of little benefit, but they may actually harm, the progress being made in the UN's SDGs.

The question that we explore in this paper is how societal and global benefits of using SIS to ensure the SDGs relate to potential difficulties, downsides and concerns in their implementation. For this purpose, we use an interpretive case study approach [3], where we analyse six empirical cases (from an original set of 10) that focus on the implementation of SIS across a range of sectors to explore how they impact the SDGs. The SDGs are internationally agreed upon goals that allow us to determine what humanity, as represented by 193 member states, finds acceptable and desirable. Measuring the impact of SIS on SDGs thus provides a way of assessing whether SIS, or an application of such a technology, is acceptable.

The benefits of SIS, as measured through their promotion of SDGs, only tells part of the story, however. In order to get a better understanding of the broader picture of the impact of these technologies, we undertake an ethical analysis of six of the cases (case studies 1, 2, 3, 4, 5, and 7) that most explicitly relate to six of the SDGs (SDGs 2, 3, 7, 8, 11, and 12). Our ethical analysis will demonstrate that, despite the potentially beneficial impact on achieving SDGs, SIS raise a number of significant ethical concerns while trying to achieve the SDGs.

While these issues are not solely confined to the use of SIS, as they can be found in issues related to many types of ICT, they are still key concerns that need to be identified in SIS use, specifically. This leads to the important question of how to balance benefits and downsides of such technologies. It shows clearly that a simple "technofix", as alluded to in the title of this paper, is not sufficient. This paper will offer a unique insight into how organisations tackle issues on the ground related to their SIS use and evaluate those issues according to the goals set out in the SDGs, which has not been empirically evaluated in a multiple-case study approach before.

The paper proceeds as follows: we start by outlining our theoretical position in a section covering our rationale for examining the use of SIS to meet the SDGs. This is followed by a description of the multiple case study approach we used in our empirical research. In our results section we describe the impact of the cases on a number of the SDGs, followed by an analysis of the ethical issues they raise. 


\section{SMART Information Systems AND A Multi-CASE StUdy APPROACH}

In this section we define the concept of Smart Information Systems (SIS) and explain why it is more suitable than the more widely used terminologies of AI and Big Data. We give a quick overview of ethical questions related to these technologies and finish by outlining our multi-case study approach.

\section{A. Smart Information Systems}

There is much discussion in academia, media and policy around AI and Big Data. One of the problems of this discussion is that the terms are poorly defined. For example, narrow AI refers to technologies that are capable of undertaking specific and clearly delineated activities whereas broad AI is a replication of general cognitive functions similar to those of humans. As these definitions are quite vague and general, one can claim that they could fall under the bracket on many other ICT applications. A similar lack of clear definition can be observed with regards to the concept of Big Data. Big Data is often defined with the help of some of its attributes, most notably volume, velocity and variety and has more recently become supplemented by the attribute of veracity and for some variability, visualization and value [4].

Our decision to use the term Smart Information Systems (SIS) is motivated by the desire to sidestep definitional problems of AI and Big Data. We use the term to denote those sociotechnical systems that make use of one particular type of AI technique, namely machine learning, usually based on artificial neural networks, to draw inferences from large amounts of typically unstructured data. By focusing on machine learning applications, we sidestep many of the social concerns and ethical issues that are associated with general AI, such as the possibility of autonomous moral agents [5] or the emergence of super intelligence [6], singularity [7] or transhumanism [8].

\section{B. Promises and Concerns of SIS}

One open question in SIS discourse refers to the criteria that could be used to determine whether an innovation or its consequences can be seen as acceptable, desirable or sustainable. At the core this is a question of universal values, ones that all those affected by an innovation could agree on. The SDGs constitute a set of internationally agreed aims that humanity, as represented in the United Nations, has agreed to pursue. The SDGs are based on clearly recognized and undisputed human needs, such as the need to end hunger, poverty or exclusion. SDGs are represented by broad and abstract aims, but these are broken down into more manageable and implementable ones. They are supported by specific and measurable targets and indicators, existing collaborations and networks and a growing amount of literature.

For the purposes of our paper the SDGs can be seen as the positive side of SIS whereas ethical concerns represent the negative side (see Figure 1). In order to promote desirable outcomes and minimise negative impacts, it is crucial to implement first steps to understand both sides. A key challenge in understanding both positive and negative aspects of these technologies is that there is very little empirical research done on them. We therefore undertook a series of ten case studies of
SIS in different application areas. The following sections provide a brief overview of the methodology of this research and then the outline of our findings.

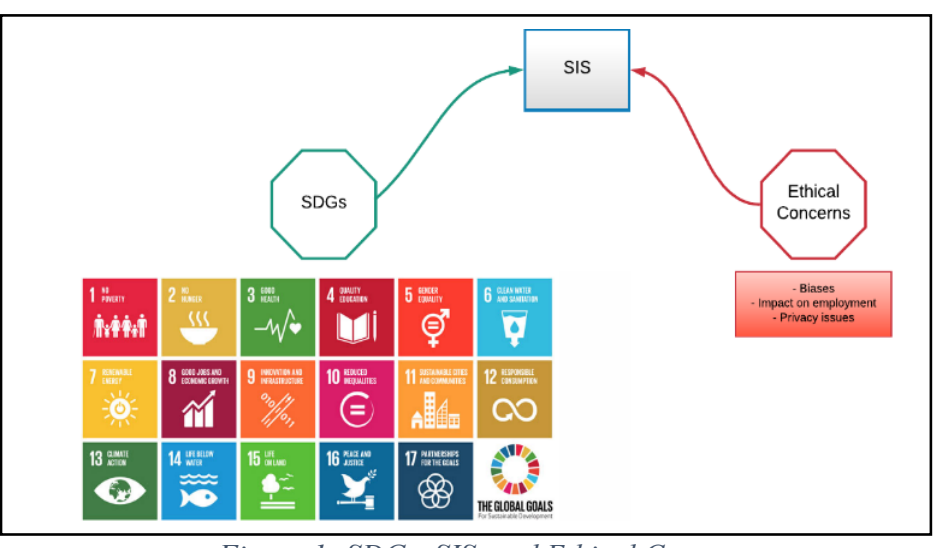

Figure 1: SDGS, SIS, and Ethical Concerns

\section{A Multiple Case Study Approach to SIS}

In order to gain a detailed understanding of the use of the technologies in their social environment, we opted for a case study approach [9]. We were interested in the lived experience of those involved in the research and therefore pursued an interpretive case study approach [3]. The structure of the case study approach was defined in a case study protocol that allowed all participants to ensure consistency and conformity of data collection and analysis [10]. During a brainstorming session, we established 16 social domains where SIS is currently being developed and used. For the purpose of this paper, we will discuss six case studies, which we undertook a literature review of ethical and social issues and a number of interviews. All interviews were held in English and transcribed. In most cases, the case referred to a single organisation. The interviews took place between June and December 2018 and took between 30 and 90 minutes each.

\section{RESULTS}

The results of our cross-case analysis provide empirical insights into how SIS is being used in a wide range of different social domains, how they are being advocated to promote and drive some of the SDGs, and also how they impact society and create their own ethical issues. Firstly, this section will outline how SIS are being used in different social domains to explicitly promote six out of the 17 SDGs (SDGs 2, 3, 7, 8, 11, and 12). These SDGs were selected as they were the most prominent goals identified in our multi-case study analysis in the use of SIS in these areas. This section will firstly demonstrate the usefulness and effectiveness of implementing SIS to meet the SDGs and the most pressing ethical issues evaluated in six out of our ten case studies (case studies 1, 2, 3, 4, 5, and 7). This section will clearly demonstrate that while SIS offer great potential and benefit to meet many societal challenges and global concerns (i.e. through the SDGs), they also pose threats and concerns to the lives of individuals and society as a whole, which need to be addressed to ensure that society benefits. 


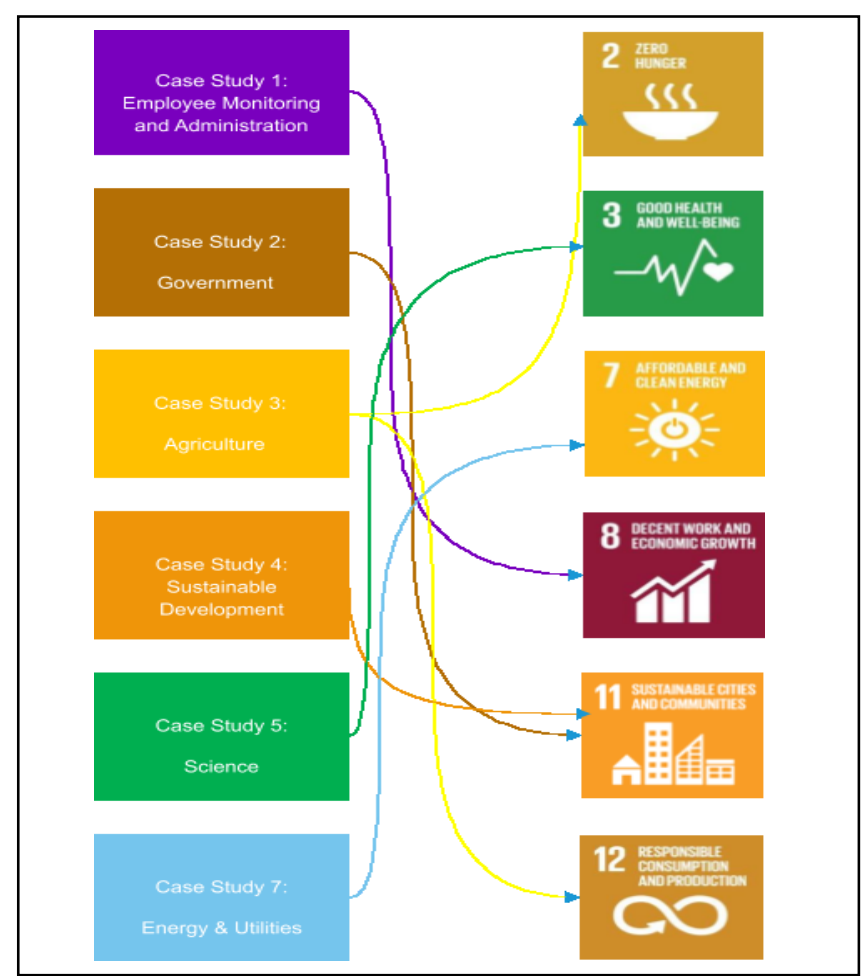

Figure 2: Mapping the Case Studies with the SDGS

The aim of SDG 2 (Zero Hunger) is to dramatically reduce undernourishment, starvation, and nutritional defects in the world; through increased agricultural produce, food security, and improvements in farming in the developing world. The UN has created a number of targets to decrease the levels of global hunger, such as doubling the 'agricultural productivity and incomes of small-scale food producers' (target 2.3) and ensuring sustainable food production systems (targets 2.4). These targets can be reached by increasing investment into technological development (target 2.A), such as SIS.

SIS is heralded as an innovative way to adapt to the challenges in a sustainable way, as it is hoped that agricultural SIS can provide data-driven answers and more efficient ways to seed, harvest, grow, and detect plant disease within the industry. Agricultural SIS has the potential to 'improve water and air quality, improved soil health, food quality and security, protection of biodiversity, improvements to quality of life, increase output, cost reductions, crop forecasting, and improved decision-making and efficiency' [11].

SDG 3 (Good Health and Well-being) aims to improve global health through areas such as maternal mortality, communicable diseases, mental health, and healthcare workforce [12]. SDG 3 aims to 'ensure healthy lives and promote well-being for all at all ages' [13]. Better health and well-being is not only viewed as a single goal for sustainable development, but is regarded as being essential for achieving all three pillars of sustainable development [14]. Health, wellbeing and sustainable development are considered to be intrinsically connected, with health regarded as a precondition indicator, as well as an outcome of successful sustainable development.
By combining the complex elements of human biology with the computational power of SIS, we can pave a path to good health and well-being. SIS supports the comparison of massive amounts of data, including individual patient health data to the greater population health data, which is crucial for determining what treatments work best for each patient. Using SIS also offers the potential to reduce development costs and bring new treatments to patients in a time-efficient manner [15].

The aim of SDG 7 (Affordable and Clean Energy) also places an emphasis on cost-efficiency and the global health of the population. Without affordable and clean energy, populations will increasingly be subject to air pollution, spending on energy that could go to health supplies and/or food, and harmful waste emissions. SDG 7 aims to ensure affordable, reliable and modern energy for all, emphasizing the need to strengthen policy in order to meet specific energy targets. Upgrading technology, such as through the use of SIS, can reduce energy consumption by $14 \%$. By 2030 , the UN aims to ensure that there is 'universal access to affordable, reliable and modern energy services', while doubling 'the global rate of improvement in energy efficiency' [16].

The expected demands on the energy sector over the coming years will be immense as a result. It is being proposed that technologies, such as SIS, used in the energy sector can help solve the Energy Trilemma: how to secure (energy security) affordable energy for all (energy equity) in a sustainable manner (environmental sustainability). The use of SIS in smart grid systems allow for renewable energy integration, deliver significant environmental benefits, and can be used as an efficient solution for energy security.

SDG 8 (Decent Work and Economic Growth) promotes the need to ensure economic growth, while acknowledging the need to resolve tensions between available jobs and the growing labour force. These tensions are exacerbated by the increasing need for technological skills in jobs, for both new and existing work positions. SDG 8 aims to increase economic productivity 'through diversification, technological upgrading and innovation', and aims to ensure 'full and productive employment and decent work for all women and men, including for young people and persons with disabilities, and equal pay for work of equal value' [16]. The use and development of SIS may help the workforce by reducing labour-intensive work, assist the processing of complex tasks, and by increasing productivity in the workplace.

SDG 11 (Sustainable Cities and Communities) attempts to achieve sustainable, resilient, safe and inclusive cities. One way to do this is by creating innovative approaches such as the adoption of SIS to 'reduce ecological harm, pollution, and injustice on the one hand; while increasing safe and affordable housing, improving infrastructure, and providing safe cities for people to live in' [17]. SIS are being proposed as a way to help achieve SDG 11 by improving mobility, reducing ecological impact, improving air quality, disaster response and economic growth [18]. SIS are being used in cities to make them 'smarter' through economic development, developing skills for the public, mobility, governance, environment and improved living standards [19]. 
SIS provide us with the opportunity to makes cities more sustainable and resilient, but they also need to incorporate responsible consumption and production of water, energy, and food, for our growing population, as advocated in SDG 12 (Responsible Consumption and Production). SIS is also playing a significant role by providing an improved understanding of consumption patterns that support devising effective environmental measures targeting specific groups such as consumers and policymakers [20]. Despite challenges posed by the use of SIS in industry, there are opportunities such as efficient and sustainable use of resources including finance, raw materials and labour that can be realised through SIS [21].

\section{Ethical Concerns with Using SIS to Meet SDGs}

While agricultural SIS offer great potential to help achieve targets in the SDGs (SDG 2 and SDG 12), there is the possibility that they may create additional ethical issues in their implementation, as demonstrated in Case study 3 - agriculture. For example, when agricultural SIS are used to provide farmers with assistance; incorrect, limited, or misleading data may lead to inaccurate recommendations and advice [22]. Interviewee 3, from case study 3 , stated that inaccurate and limited data was the main cause for poor or ineffective recommendations, rather than flaws with their algorithms. Issues relating to flawed data is also exacerbated by farmers with poor record-keeping, an inability to use SIS, or implement these recommendation. Inaccurate recommendations, resulting from SIS, may cause poor harvests, harm to crops and livestock, and damage to the farmer's business.

A challenge for effective agricultural SIS use is that most farming is done on small farms or in LMICs (low-to-middleincome countries) with low technological advancements; whereas, current agricultural SIS use is on large monoculture farms [23][24]. In order to meet SDG 2 and SDG 12, agricultural SIS should be affordable, usable, and accessible to LMIC farmers in an economically sustainable way [25][26][27]. The interviewees from case study 3 reiterated this sentiment, stating that if SIS is not economically affordable and beneficial to the farmer, they will not be adopted. To meet these goals, farmers are forced to 'supply cheaper, more diverse, and quicker produce' [11].

There is a possibility that agricultural SIS will actually exacerbate inequalities, rather than prevent them, which is in contrast to target 12.C - reducing harm to poor and disadvantaged communities [28]. The retrieval of farm data may cause privacy infringements, particularly in LMICs where there is little data regulation and protection [29]. There is also the possibility that farmers may lose control of their farm because companies, such as John Deere, are preventing farmers from tampering with their machinery, which contains SIS, on intellectual property grounds [30][31]. Farmers are also concerned that their data may be leaked or given to thirdparties, making them sceptical about adopting SIS [32]. Their data may be used against them by commodity traders, governmental bodies, or competitors, so they need to be confident that their data will be protected 'from misuse, hacking, and the misappropriation for economic or marketing purposes' [11].

Agricultural SIS may also provide recommendations that do not take into account effects on land external to the farm being analysed, which could lead to harmful runoff, habitat damage, and pollution [33]. Countries have varying sustainability standards, so it is difficult for SIS to accurately take these into account: 'Different algorithms are required because of the varying climatic conditions, crop types, and needs of farmers worldwide' (in press). In addition to sustainability metrics, SIS may 'upset, injure or even kill livestock and/or local wildlife. Robots, sensors and unmanned aerial vehicles (UAVs) also have the potential to emit toxic material, fumes and waste into their surrounding environment' [11], causing harm to the health of animals, humans, and communities in the surrounding areas.

Case study 5 was used to understand some of the ethical concerns that arise from the use of SIS in health, specifically, health-related issues that affect the brain and how they could be treated. The organisations interviewed used SIS to build a research infrastructure aimed at the advancement of neuroscience, medicine and computing. Results from the case study indicate that the main ethical concerns are privacy and confidentiality. There is a risk of identifying patients because hackers could access patient data. The data could be reidentified to the primary source [34], thus violating their privacy, and potentially being used to harm the individual subsequently.

Security at the software level is an issue when using health SIS. With the use of the internet, the systems are opening ports into hospitals which means that there should be a lot of safeguards for specific parts of a specific server [35]. There was also a concern in case study 5 about discrimination and bias resulting from the use of health SIS and the issue of transparency of the processes that are involved in research used to understand diseases and treatments. The use of SIS in promoting health also has implications associated to the availability of resources which could result in a digital divide between those who have the resources to use most of the SIS platforms and those who cannot, an issue that was also addressed in Case study 7 - energy \& utilities.

The use of SIS systems in energy distribution hold the promise that countries will be able to ensure affordable and sustainable energy for the ever-increasing energy demands of smart living, it also presents a number of ethical challenges, which were identified in our case study 7 - energy \& utilities. This case study explored ethical issues that occur in the use of SIS in the energy sector. According to the interviewees, the current barrage of GDPR articles in the media has raised the public's privacy concerns and suspicion towards the company and the use of SIS in the energy industry. The company, in case study 7, was vocal about addressing issues of social acceptability of smart meters and privacy concerns that the enduser may have. For example, it has coordinated the development of a code of conduct to address public concerns and sought to have it approved by the Personal Data Authority 
to ensure that the company remains within the law and attracts public trust.

Another major concern identified in case study 7 related to issues around cybersecurity. As a result of the complexity of the decentralised architecture, and the digitisation of multiple points in the grid, there is a concern that they can be individually attacked to trigger a cascading response, leading to energy disruptions or failure of the infrastructure (e.g., blowing the fuses of energy exchanges). As it will be impossible to safeguard the infrastructure entirely, the emphasis is shifting towards containing possible contagion and its cascading effects. Specific cyber threats and implications for cybersecurity are difficult to predict in order to make provisions into the system design and the institutional environment, yet a concerted effort has been put in place through a pan-European Cybersecurity Act to protect the management of critical infrastructures, equipment and consumer products.

In many businesses, SIS are being used as a means to deliver enhanced customer service and improved business management procedures. By using SIS to monitor business operations, through tracking-capable software, businesses are, for instance, able to track products but also to monitor employees. Case study 1 (employee monitoring and administration) focused on an international company that develops SIS tracking equipment for the purpose of deducing how assets are used in order to either bill according to their usage, or to identify usage fraud. The case study examined IoTbased SIS that makes use of data collection and manipulation to support monitoring and tracking in businesses.

The most prominent ethical issues that arose in case study 1 were the possibility for malicious use, privacy infringements, and the responsibility, transparency and trust required by the organisation using these technologies. A measure to safeguard many of these issues from occurring was ensuring accurate informed consent was granted. Providing the opportunity to stakeholders to consent to the collection, manipulation, or deletion of data is very significant to ensuring data protection. Nevertheless, even though the technology provides for features that can encourage ethical use of the system, the possibility for system abuse cannot be totally excluded.

One of the overriding concerns identified in the case study focus on how to ethically design employee monitoring software for other companies to use with their own assets and resources. Design with respect to access controls is therefore important as well as the issue of consent. It is not unusual that several of the identified ethical issues interconnect for a particular SIS, and it is quite important to be cautious with the handling of data across the hierarchy of system users. This strongly correlates with ensuring the SDG 8 is met, namely to promote good work environments for people around the world.

SIS offers great benefit to the public sector to ensure sustainable cities and communities, but may also create their own ethical concerns as a result, which were identified in case study 2 (government) and case study 4 (sustainable development). Municipalities benefitting from the development and use of SIS need to ensure that they work effectively. One way to ensure this is through the provision of sufficient, dynamic, and rich data. In the case studies, interviewees emphasised the importance of retrieving and using accurate datasets for successfully running their SIS. If the algorithms do not have sufficient training data, then the recommendations provided may be misleading or inaccurate.

If there are issues with the accuracy of data, it may misrepresent the city and its inhabitants [18]. There is a threat that SIS may compartmentalise cities, reducing their complexity and richness, which may lead to harmful or biased recommendations and policy [36]. With the increased integration of SIS in cities, there is also a threat of a digital divide at different levels. There is the possibility that rural areas will get left behind as a result of increased technological development within cities; some areas and citizens within cities may benefit from SIS, while others are disadvantaged; certain cities (such as capitals) may receive far greater SIS investment and development that other cities; and there may also be a greater digital divide and resulting inequalities between developing and developed nations who can or cannot afford to implement these technologies.

Another concern about the increased digitalisation of the city infrastructure is the increased vulnerability to malicious hacking, stolen data, disruption of systems within the city, or privacy infringements [37][38]. Privacy was an issue raised in all five organisations in these two case studies. Interviewee 1, from case study 4, stated that whenever data is collected about citizens, their privacy should be protected. Whenever cities have access to citizens' data, there is a threat that it will be used for surveillance purposes. Also, with the increased integration of private organisations in SIS public projects, there is also a threat that they will use this data for illegitimate purposes.

There is a concern that private organisations will prioritise their interests in public-private SIS projects and push a technologizing approach, which may not be in the best interests of the city or its citizens: 'Corporations are providing advice, guidance and implementing technologies within cities, and this may not be done impartially or in the best interests of the city' [17]. Cities may become dependent on private SIS companies, which may lead to 'technological lock-in', thus jeopardising a municipality's self-governance. The interviewee from case study 2 stated that obtaining data from third-party organisations often incurred substantial costs, but that their data often far surpassed publicly-available data, thus necessitating this partnership. However, there is a concern that '[i]f corporations are heavily involved with any SIS government project, the city may become overly dependent on those corporations, putting public decision-making and governance in jeopardy' [39].

Most of the public servants working on SIS projects indicated that they were aware of this threat and many expressed that they tried to initiate a data sovereign approach, if possible, and were cautious to avoid technological lock-in with private SIS companies. Some were concerned about the 
high costs required for investing in SIS projects. While technologically-savvy cities may encourage national and foreign investment, there are no guarantees that a city will see a return on their SIS investment. For example, interviewee 3 from case study 4, stated that their SIS project was loss-making and would have been terminated earlier if run by a company.

\section{CONCLUSION}

The importance of SIS in society will continue to grow in the future, particularly when meeting the SDGs. The first step towards the effective use of SIS to meet the SDGs is to acknowledge potential issues and identify ways to ensure that society benefits, while reducing harms, from their use. "Technofixing" the SDGs, through the ill-thought-through use of SIS, may create a range of ethical issues, which have been outlined in this paper using an interpretivist multi-case study analysis. Six SDGs $(2,3,7,8,11,12)$ were examined to extrapolate beneficial aspects of using SIS, while also identifying ethically problematic issues. Domain-specific literature was analysed and contrasted with what is being used to gain some empirical insights regarding the ethical issues that relate to the use of SIS. In the discussion section, the paper evaluated the main benefits and drawbacks of using SIS for those six SDGs and proposed steps that can be implemented to ensure their ethical use.

\section{REFERENCES}

[1] B. C. Stahl and D. Wright, "Ethics and privacy in AI and Big Data: Implementing responsible research and innovation", IEEE Security \& Privacy, vol. 16, issue 3, pp. 26-33, 2018.

[2] M. Chui, M. Harryson, J. Manyika, R. Roberts, R. Chung, A. Heteren, and P. Nel, "Notes from the AI frontier: Applying AI for social good", McKinsey Global Institute, 2018.

[3] G. Walsham, G. "Interpretive case studies in IS research: nature and method", European Journal of Information Systems, vol. 4, issue 2, pp. 74-81, 1995, https://doi.org/doi:10.1057/ejis.1995.9I.

[4] M.F. Uddin and N. Gupta, "Seven V's of Big Data: Understanding Big Data to extract value", in Proceedings of the 2014 Zone 1 Conference of the American Society for Engineering Education, IEEE, pp. 1-5, 2014.

[5] C. Allen, "Artificial life, artificial agents, virtual realities technologies of autonomous agency", in L. Floridi (Ed.), The Cambridge Handbook of Information and Computer Ethics, Cambridge University Press, pp. 219233, 2010.

[6] S. Torrance, "Super-intelligence and (super-)consciousness", International Journal of Machine Consciousness, vol. 4, issue 2, pp. 483501, 2012. https://doi.org/10.1142/S1793843012400288

[7] R. Kurzweil, The Singularity is Near, London: Gerald Duckworth \& Co Ltd, 2006.

[8] D. Livingstone, Transhumanism: The History of a Dangerous Idea, CreateSpace Independent Publishing Platform, 2015.

[9] J.D. Farquhar, Case Study Research for Business, Sage Publications Ltd, 2012.

[10] R. K. Yin, Case Study Research: Design and Methods (Third Edition). Sage Publications, Inc, 2003.

[11] M. Ryan, "Ethics of using AI and Big Data in agriculture", ORBIT Journal, 2019, in print.

[12] Y.M. Asi, and C. Williams, "The role of digital health in making progress toward Sustainable Development Goal (SDG) 3 in conflict-affected populations", International Journal of Medical Informatics, vol. 114, pp.114-120, 2018.

[13] A.R. Nunes, K. Lee, and T. O'Riordan, "The importance of an integrating framework for achieving the Sustainable Development Goals: the example of health and well-being", BMJ Global Health, 1(3), 2016.
[14] P.S. Hill, K. Buse, and Brolan CE, "How can health remain central post2015 in a sustainable development paradigm?", Global Health, vol. 10, issue 18, 2014.

[15] D. Novillo-Ortiz, H.D.F. Marin, and F. Saigi-Rubio, The role of digital health in supporting the achievement of the Sustainable Development Goals (SDGs), 2015.

[16] United Nations, "Sustainable development goals - United Nations", United Nations Sustainable Development [website], 2018.

[17] M. Ryan and A. Gregory, "Ethics of using smart city AI and Big Data: The case of four large European cities", ORBIT Journal, 2019, in print.

[18] R. Kitchin, "Data-driven networked urbanism", The Programmable City Working Paper 14, 2015.

[19] V. Albino, U. Berardi, and R.M. Dangelico, "Smart cities: Definitions, dimensions, performance, and initiatives", Journal of Urban Technology, vol. 22, issue 1, pp. 3-21, 2015.

[20] A. Froemelt, D.J. Dürrenmatt, and S. Hellweg, "Using data mining to assess environmental impacts of household consumption behaviors", Environmental Science \& Technology, vol. 52, issue. 15, pp. 8467-8478, 2015. https://doi.org/10.1021/acs.est.8b01452

[21] J.M. Müller, D. Kiel, and K.I. Voigt, "What drives the implementation of industry 4.0? The role of opportunities and challenges in the context of sustainability", Sustainability, vol. 10, issue 1, 2018. https://doi.org/10.3390/su10010247

[22] L. Taylor, and D. Broeders, "In the name of development: Power, profit and the datafication of the global south", Geoforum, vol. 64, pp. 229-37, 2015.

[23] I. Carbonell, "The ethics of Big Data in big agriculture", Internet Policy Review, vol. 5, issue 1, pp. 1-13, 2016.

[24] USDA NASS, Census of Agriculture Highlights: Farm Economics, 2014.

[25] E.M. Micheni, "Diffusion of Big Data and analytics in developing countries", The International Journal of Engineering and Science, vol. 4, issue 8, pp. 44-50, 2015.

[26] R. Panicker, "Adoption of Big Data technology for the development of developing countries", Proceedings of National Conference on New Horizons in IT-NCNHIT, 2013.

[27] UN Global Pulse, Big Data for Development: Challenges \& Opportunities, Naciones Unidas, Nueva York, mayo, 2012.

[28] K. Poppe, S. Wolfert, and C. Verdouw, "How ICT is changing the nature of the farm: A research agenda on the economics of Big Data", 11th European IFSA Symposium, Farming Systems Facing Global Challenges: Capacities and Strategies, Proceedings, Berlin, Germany, 14 April 2014, 2014.

[29] L. Taylor, "Safety in numbers? Group privacy and Big Data analytics in the developing world", in L. Taylor, B. van der Sloot, and L. Floridi, Group Privacy: The Challenges of New Data Technologies, Springer, pp. 13-36, 2017.

[30] M. Carolan, "Publicising food: Big Data, precision agriculture, and co experimental techniques of addition”, Sociologia Ruralis Vol. 57, Issue 2, pp. 135-54, 2017.

[31] S. Wolfert, "Big Data in smart farming-a review", Agricultural Systems vol. 153, pp. 69-80, 2017.

[32] J.L. Ferris, "Data privacy and protection in the agriculture industry: Is federal regulation necessary", Minn. JL Sci. \& Tech, vol. 18, issue 1, pp. 309-342, 2017.

[33] K. Kosior, "Agricultural education and extension in the age of Big Data", European Seminar on Extension and Education, 2017.

[34] K.S. Rommelfanger, et al, "Neuroethics questions to guide ethical research in the international brain initiatives", Neuron, vol. 100, pp. 19 36, 2018. https://doi.org/10.1016/j.neuron.2018.09.021

[35] P.J. Bentley, M. Brundage, O. Häggström, T. Metzinger, "European parliament, European parliamentary research service, scientific foresight unit", Should We Fear Artificial Intelligence?: In-depth Analysis, 2018.

[36] S. Sholla, R. Naaz, and M.A. Chishti, "Ethics aware object-oriented smart city architecture", China Communications, vol. 14, issue 5, pp. 160-173, 2017.

[37] M. Batty, K.W. Axhausen, F. Giannotti, A. Pozdnoukhov, A. Bazzani, M. Wachowicz, G. Ouzounis, and Y. Portugali, "Smart cities of the future", 
The European Physical Journal Special Topics, vol. 214, issue 1, pp. 481518, 2012.

[38] R. Kitchin, T.P. Lauriault, and G. McArdle, "Smart cities and the politics of urban data", Smart Urbanism: Utopian Vision or False Dawn? Routledge, London, pp. 16-33, 2015. ISBN 9781138844223.
[39] M. Ryan, "Ethics of public use of AI and Big Data", ORBIT Journal, vol. 2, issue 2, 2019. 